\title{
Management of Second Wave of COVID 19 with Ledipasvir / Sofosbuvir Combination. Will it Work? A Review Article
}

\author{
Sara A. Raslan ${ }^{2}$, Eslam M. Shehata ${ }^{2}$, Mohamed Raslan ${ }^{1,2}$, Nagwa A. Sabri ${ }^{1,3 *}$ \\ ${ }^{1}$ Department of Clinical Pharmacy, Faculty of Pharmacy- Ain Shams University, Cairo, Egypt \\ ${ }^{2}$ Drug Research Centre, Cairo, Egypt \\ ${ }^{3}$ Principal Investigator of Drug Research Centre, Cairo, Egypt
}

\author{
DOI: $10.36348 /$ sjmps.2020.v06i10.003 \\ | Received: 13.09.2020 | Accepted: 21.09.2020 | Published: 29.10.2020 \\ *Corresponding author: Nagwa Ali Sabri
}

Abstract

The COVID-19 epidemic has already exerted a wide effect on the global level and the rapid growth of infected cases resulted in an overwhelmed health care providing systems. Many countries that have already peaked and flattened the infection curve reached now the minimum infection rate and minimal or zero reporting of new cases. As a consequence, many countries are reducing their precautionary measures which might lead to the emerging of a second incidence wave of SARS-CoV-2. On the other hand, direct antiviral combination therapy as Ledipasvir/Sofosbuvir showed sufficient efficacy in treatment of chronic hepatitis $\mathrm{C}$ virus (HCV) with an acceptable safety profile showing a minimum side effect and well tolerated upon administration during the treatment time course as well. Besides, according to published studies and collected data, this combination recorded a minimal drug interactions with the other therapeutic agents used for the management of co-morbid conditions with COVID-19. Finally, the reported results of performed studies showed a superior time to clinical recovery from COVID-19 for Sofosbuvir / Daclatasvir regmin over that of Lopinavir/ritonavir, and thus, the proposal of involving Ledpasvir/Sofosbuvir combination as a better treatment option than other direct antiviral agents in the management of COVID-19 for the second wave.

Keywords: COVID-19, Second wave, Ledipasvir/Sofosbuvir, hepatitis C virus, Darunavir.

Copyright (C) 2020 The Author(s): This is an open-access article distributed under the terms of the Creative Commons Attribution 4.0 International
License (CC BY-NC 4.0) which permits unrestricted use, distribution, and reproduction in any medium for non-commercial use provided the original
author and source are credited.

\section{INTRODUCTION}

Corona virus Disease-2019 (COVID-19) has emerged as a rapidly spreading infective disease worldwide. This disease is caused by Severe Acute Respiratory Syndrome-Coronavirus-2 (SARS-CoV-2). Disease spreading occurred primarily through large respiratory droplets, despite the fact that possibility of other routes of transmission cannot be ruled out, as the virus has been found in feces and urine of affected individuals [1]. The disease severity has varied from mild self-limiting flu-like illness to severe pneumonia, respiratory faliure and death.

It was found that about $21,294,845$ confirmed cases of COVID-19 worldwide with a death rate of $3.57 \%$ according to the situation report of World Health Organization on August 16, 2020 [2].

It is worthy to mention that, the number of unconfirmed and unreported cases is probably much higher than the reported cases and the actual mortality may be less than $1 \%$ which is similar to that of severe seasonal influenza [3]. Different continentals showed different prevalence percentages for example; Africa showed a 945,165 confirmed cases with a mortality rate of $1.95 \%$, Europe showed a 3,754,649 confirmed cases with a mortality rate of $5.7 \%$, USA showed a $11,420,860$ confirmed cases with a mortality rate of $3.6 \%$ till 26th June, 2020 [2].

The understanding of the epidemiological characteristics of this infection is evolving on a daily basis as the disease is spreading to different parts of the globe. A historical review suggested that the highest mortality rate in Spanish Flu pandemic in 1918 was also related to the second wave of the disease [4], where, the Spanish Flu remained spreading for two years, in consecutive waves of infection around the world [5].

Assumptions about COVID-19 disease that there is no disease recurrence after the first wave of the disease proved to be misconception leading to ignorance of people to the necessary protective measures and, consequently, engaged in some misbehavior, such as mixing socially with others or 
holiday outings. These social conditions will dramatically increase the demand on medical staff and health care services and jeopardize lives of many people globally [6].

When the prevalence of SARS-CoV-2 has decreased for some time, the expected result was social reduction or elimination of protective measures. This was obvious in the increase in social relations and travel trips, people noncompliance with health precautions, non-compliance with social distance, and the lack of governmental serious actions in many countries, resulted in high probability of a second wave of corona virus spread. When the imposed restrictions were reduced in April and reopening of normal social and economic behavior in society occurred, the disease came back again. This was the case of Iran in which the second wave started from May till now [6].

Ledipasvir (LDV) is an antiviral drug aimed at a protein (NS5A) in the hepatitis $\mathrm{C}$ virus. As this protein is involved in viral replication, ledipasvir will reduce the amount of virus in infected patients. Sofosbuvir (SOF) is a nucleotide analogue antiviral drug that is used in combination with other drugs to treat chronic hepatitis $\mathrm{C}$ virus (HCV). As the effectiveness of regimens containing interferon can be limited by adverse effects, there is interest in studying other drugs to use in combination with sofosbuvir [7].

Both drugs are available in LDV/SOF combination and is marketed in the form of tablets as a Ledipasvir $90 \mathrm{mg}$ and Sofosbuvir $400 \mathrm{mg}$, under brand name HARVONI ${ }^{\circledR} 90 \mathrm{mg} / 400 \mathrm{mg}$ Tablets [8]. Ledipasvir and Sofosbuvir are indicated for the treatment of chronic hepatitis $\mathrm{C}$ virus (HCV) genotype 1 in adults [9].

The advantage of ledipasvir/sofosbuvir lies in its once-daily fixed-dose regimen that does not require the coadministration of ribavirin [10]. Compared to the previous standard of care requiring treatment for 48 weeks, duration of chronic HCV-1 infection treatment with LDV/SOF is just 12 or 24 weeks, as recommended by EASL, American Association for the Study of Liver Diseases, and Infectious Diseases Society of America $[11,12]$. Among treatment-naïve (TN) patients with $\mathrm{HCV}$ RNA $<6$ million IU/mL without cirrhosis, treatment duration can even be reduced to 8 weeks [13$15]$.

\section{DISCUSSION}

Compared to the first generation of $\mathrm{HCV}$ protease inhibitors, the LDV/SOF combination has the advantage few drug interactions, thus, leading to a wide range of prescription possibilities among $\mathrm{HCV}-1$ or -4infected patients with comorbidities [16]. However, coadministration with amiodarone, carbamazepine, phenytoin, rifampicin, rosuvastatin, St John's wort, tipranavir, and simeprevir should be avoided, while coadministration with digoxin, dabigatran etexilate, and TDF requires a close monitoring [17-19].

LDV and SOF are substrates of P-glycoprotein transporters [20, 21], inducers of P-glycoprotein as rifampicin, St John's wort, carbamazepine, phenytoin, and tipranavir may decrease intestinal absorption of LDV/SOF leading to decreased effcacy. Other inducers potentially associated with signifcant interaction with LDV/SOF include digoxin, dabigatran etexilate, rosuvastin, simeprevir, and tenofovir disoproxil fumarate when associated with pharmacologic enhancers such as ritonavir [22, 23].

LDV solubility decreases as $\mathrm{pH}$ increases, so that drugs that increase gastric acidity (eg, antacids, proton pump inhibitors) may decrease plasma concentrations of LDV. Proton pump inhibitors should not be taken before LDV/SOF. Twenty milligrams of proton pump inhibitor equivalent to omeprazole $20 \mathrm{mg}$ can be safely administrated at the same time or $>2$ hours after LDV/SOF [24, 25].

It was proven that Sofosbuvir, the clinically approved anti-hepatitis $\mathrm{C}$ virus (HCV) drug, is capable of suppressing other families of positive-strand RNA viruses; Flaviviridae and Togaviridae. Coronaviruses are a family of positive-strand RNA viruses with conserved polymerase, so SARS-CoV-2 RdRp is very likely to be effectively inhibited by Sofosbuvir [26]. Also, it was reported that (ledipasvir/sofosbuvir) could be attractive drugs that can inhibit $3 C$-like protease of the virus and treat COVID-19 with minimal side effects [27].

Sofosbuvir is an approved direct antiviral therapy for $\mathrm{HCV}$ and is effective against other types of positive-strand RNA viruses. Corona viruses are a family of positive-strand RNA viruses with conserved polymerase, so SARS-CoV-2 RdRp is most probably to be effectively inhibited by sofosbuvir which is safe and well tolerated at $400 \mathrm{mg}$ daily in a 24 -week therapeutic regimen. Besides, its active metabolite shows a high degree of intracellular stability which leads to a proposal that SARS-CoV-2 infection could also likely to be cured by sofosbuvir. Also, it was reported that sofosbuvir does not affect the main cytochromal metabolizing enzymes as cytochrome P450 system [28].

The superiority of using Ledipasvir / sofosbuvir to interferon / ribavirin combination as a treatment for patients with HCV genotype 4 for a period of 12 weeks was well tolerated and resulted in $100 \%$ sustained virological response (SVR) for all patients with irrespective of previous treatment status and underlying liver fibrosis [29]. 
Regarding antiviral activity, the antiviral activity of LDV was tested against viruses other than $\mathrm{HCV}$ in cell based assay systems. These viruses include five closely related viruses (bovine viral diarrhea virus [BVDV], West Nile virus, yellow fever virus, dengue virus, and Banzi virus), as well as six unrelated viruses, including respiratory syncytial virus (RSV), human rhinovirus, influenza A virus, influenza B virus, hepatitis B virus (HBV), and HIV. LDV showed active antiviral activity against $\mathrm{HCV}$ with an EC50 of 0.004 nM. In contrast, EC50 values against the 11 other viruses were $>10,000 \mathrm{nM}$, indicating that LDV is highly selective for $\mathrm{HCV}$ compared with other tested viruses [30].

Table-1: Current versus Proposed Management Protocols for Second Wave of COVID-19 Containing SOF/LED Combination

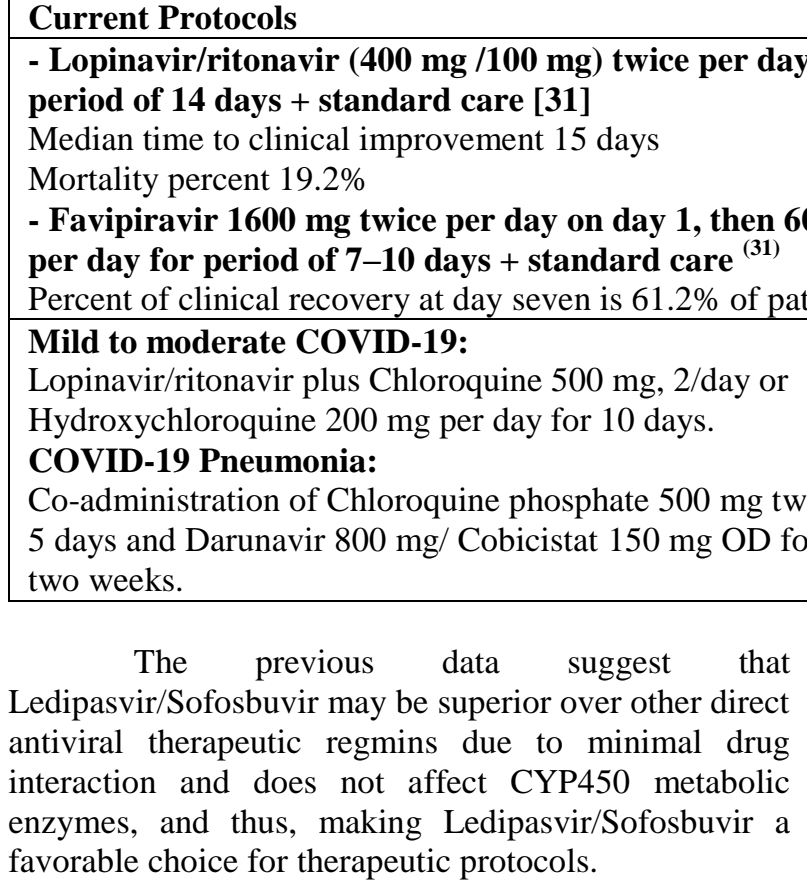

\section{CONCLUSION}

From the aforementioned review, it can be concluded that an expected second COVID-19 wave including a higher incidence and mortality rates than the first one is most likely to occur as a result of large category of population attitudes of non-compliance to protective measures, on the other hand, the healthcare services expected to be overwhelmed.

Concerning management protocols used and direct antivirals included in these protocols, it was clear that different direct antiviral agents showed several drug interactions with other therapeutic agents used for treatment of some co-morbid conditions as diabetes compared to Ledipasvir / Sofosbuvir combination which proved effectivenesss, safety and tolerability in several cases and thus can be superior to the other direct antivral agents reported in the management of COVID19.

\section{REFERENCES}

1. Del Rio, C., \& Malani, P. N. (2020). COVID-19New Insights on a Rapidly Changing Epidemic. JAMA, 323(14):1339-1340.

2. Coronavirus disease 2019 (COVID-19) Situation Report - 209. https://www.who.int/docs/default-

\author{
Proposed Protocols \\ Sofosbuvir / Daclatasvir (400/60mg) + \\ standard care for a period of 14 days [32] \\ No of patients Clinically recovered in less \\ than 14 days are 29 patients out of 33 patients \\ $(87 \%)$. \\ Time to clinical recovery 6 days in median \\ (range from 4 to 10 days). \\ Ledipasvir / Sofosbuvir (90/400mg) \\ Less or no interactions with other therapeutic \\ agents like Lopinavir/ritonavir or \\ Hydroxychloroquine
}

\section{Can be used safely with antidiabetic agents.}


Recommendations for Testing, Managing, and Treating Hepatitis C. Available from: http://www.hcvguidelines.org/full-report/uniquepatient populations -patients-hivhcv coinfection. Accessed April 6, 2015.

12. EASL Recommendations on Treatment of Hepatitis C 2015. J Hepatol. 63(1):199-236, 2015.

13. Fazel, Y., Lam, B., Golabi, P., \& Younossi, Z. (2015). Safety analysis of sofosbuvir and ledipasvir for treating hepatitis C. Expert opinion on drug safety, 14(8), 1317-1326.

14. Jacobson, I., Kwo, P., Kowdley, K., Yang, J., Zhu, Y., Hyland, R., ... \& Afdhal, N. (2014). Virologic Response Rates to All Oral Fixed-Dose Combination Ledipasvir/Sofosbuvir Regimens Are Similar in Patients With and Without Traditional Negative Predictive Factors in Phase 3 Clinical Trials: 1945. Hepatology, 60. (4 Suppl):1141A1142A.

15. Gentile, I., \& Borgia, G. (2014). Ledipasvir/Sofosbuvir administration achieves very high rate of viral clearance in patients with HCV genotype 1 infection without cirrhosis, regardless of ribavirin co-administration or length of treatment. BMJ Evidence-Based Medicine, 19(6), 223-224.

16. Nkuize, M., Sersté, T., Buset, M., \& Mulkay, J. P. (2016). Combination ledipasvir-sofosbuvir for the treatment of chronic hepatitis $\mathrm{C}$ virus infection: a review and clinical perspective. Therapeutics and clinical risk management, 12, 861 .

17. Harvoni (ledipasvir and sofosbuvir) tablets for oral use [US package insert]. Foster City, CA: Gilead Sciences Inc; 2015.

18. German, P., Pang, P., Fang, L., Chung, D., \& Mathias, A. (2014). Drug-Drug Interaction Profile Of The Fixed-Dose Combination Tablet Ledipasvir/Sofosbuvir: 1976. Hepatology, 60(4 Suppl):1162A.

19. German, P., Garrison, K., Pang, P. S., Stamm, L., Ray, A., \& Shen, G. (2015, February). Drug-drug interactions between anti-HCV regimen ledipasvir/sofosbuvir and antiretrovirals. In Conference on retroviruses and opportunistic infections (pp. 23-26).

20. Gritsenko, D., \& Hughes, G. (2015). Ledipasvir/Sofosbuvir (harvoni): improving options for hepatitis $\mathrm{C}$ virus infection. Pharmacy and Therapeutics, 40(4), 256.

21. Keating, G. M. (2015). Ledipasvir/Sofosbuvir: a review of its use in chronic hepatitis C. Drugs, 75(6), 675-685.

22. Gentile, I., Buonomo, A. R., Zappulo, E., Coppola, N., \& Borgia, G. (2014). GS-9669: a novel non-nucleoside inhibitor of viral polymerase for the treatment of hepatitis $\mathrm{C}$ virus infection. Expert Review of Anti-infective Therapy, 12(10), 1179-1186.
23. German, P., Mathias, A., Yang, J., McNair, L., Shen, G., Vimal, M., ... \& Lawitz, E. (2013). The Pharmacokinetics of Ledipasvir, an HCV Specific NS5A Inhibitor in HCV-Uninfected Subjects with Moderate and Severe Hepatic Impairment: 467. Hepatology, 58.

24. Kirby, B., German, P., \& Sheen, G. (2013). No differences in the pharmacokinetics of sofosbuvir (GS-7977) and sofosbuvir/ledipasvir (GS7977/GS-5885) fixed dose combination between Japanese and Caucasian [Abstract 954]. Hepatol Int, 7(Suppl 1), S375-6.

25. German, P., Garrison, K., Pang, P. S., Stamm, L., Ray, A., \& Shen, G. (2015, February). Drug-drug interactions between anti-HCV regimen ledipasvir/sofosbuvir and antiretrovirals. In Conference on retroviruses and opportunistic infections (pp. 23-26).

26. Sayad, B., Sobhani, M., \& Khodarahmi, R. (2020). Sofosbuvir as repurposed antiviral drug against COVID-19: why were we convinced to evaluate the drug in a registered/approved clinical trial?. Archives of medical research.

27. Li, C., Wang, L., \& Ren, L. (2020). Antiviral mechanisms of candidate chemical medicines and traditional Chinese medicines for SARS-CoV-2 infection. Virus research, 198073.

28. Sayad, B., Sobhani, M., \& Khodarahmi, R. (2020). Sofosbuvir as repurposed antiviral drug against COVID-19: why were we convinced to evaluate the drug in a registered/approved clinical trial?. Archives of medical research.

29. Kohli, A., Kapoor, R., Sims, Z., Nelson, A., Sidharthan, S., Lam, B., ... \& Sugarman, K. (2015). Ledipasvir and sofosbuvir for hepatitis C genotype 4: a proof-of-concept, single-centre, open-label phase 2a cohort study. The Lancet Infectious Diseases, 15(9), 1049-1054.

30. Cheng, G., Tian, Y., Doehle, B., Peng, B., Corsa, A., Lee, Y. J., ... \& Dvory-Sobol, H. (2016). In vitro antiviral activity and resistance profile characterization of the hepatitis $\mathrm{C}$ virus NS5A inhibitor ledipasvir. Antimicrobial agents and chemotherapy, 60(3), 1847-1853.

31. Teoh, S. L., Lim, Y. H., Lai, N. M., \& Lee, S. W. H. (2020). Directly Acting Antivirals for COVID19: Where Do We Stand? Front Microbiol. 11:1857.

32. Sadeghi, A., Asgari, A. A., Norouzi, A., Kheiri, Z., Anushirvani, A., Montazeri, M., ... \& Radmard, A. R. (2020). Sofosbuvir and daclatasvir compared with standard of care in the treatment of patients admitted to hospital with moderate or severe coronavirus infection (COVID-19): a randomized controlled trial. Journal of Antimicrobial Chemotherapy. 